\title{
Fuzzy Shortest Path Problem by Using Ranking Function
}

\author{
S. Ramkumar ${ }^{1}$, Dr. M. Ananthanarayanan ${ }^{2}$ \\ ${ }^{1}$ Assistant Professor, P.G.Dept of Mathematic, A.M.Jain College, Tamil Nadu India \\ ${ }^{2}$ Associate Proessor ,P.G.Dept of Mathematics, A.M.Jain College, Tamil Nadu India
}

\begin{abstract}
In this paper algorithm is presented to find shortest path in fuzzy environment. The Trapezoidal fuzzy numbers is given to assess the shortest path from initial node to terminal node by using Ranking Functions.
\end{abstract}

Keywords: Fuzzy set theory, Trapezoidal fuzzy numbers, Ranking functions

\section{Introduction}

The shortest path problems deals on finding the path with minimum distance. It is one of the most fundamentals and well known optimization problems that appear in many application as a sub-problem..In shortest path problems it is assumed that decision maker is certain about the parameters such as distance, days, time etc., between different nodes.

Many researchers have developed much concentration to the fuzzy shortest path, since I is essential to many of applications [1],[2] [6-9]. In some applications the numbers associated with nodes (edges) may represent characteristics (parameters).

The shortest path (length) was first developed by Dubois and Prade [3] Takahashi Yamakami [4] discussed the shortest path with fuzzy parameters.In real life situations there always exist uncertainty about the parameters between different nodes. In such cases the parameters are represented byfuzzyNumbers(Liu.ST \& Kao). (10).

\section{Preliminaries}

Some basic definitions are reviewed

\subsection{Definition}

The characteristic function $\mu_{\mathrm{A}}$ of a set $\mathrm{A}$ is subset of $\mathrm{X}$ assigns a value 0 or 1 to each element of $X$. this functions can be generalized to a function such that the value assigned to the element of the Universal set $\mathrm{X}$, fall within a specified range $[0,1]$. The assigned value indicate membership function of the element in the set A.

2.2 The functions $\boldsymbol{\mu}_{\mathrm{A}}$ is called the membership function and the set $A=\left\{\left(\mathrm{x}: \boldsymbol{\mu}_{\mathrm{A}}(\mathrm{x})\right\}\right.$ defined by $\boldsymbol{\mu}_{\mathrm{A}},(\mathrm{x} \mathrm{x})$ is called a fuzzy set.

2.3 A fuzzy number $A=(a, b, c, d)$, is a trapezoidal fuzzy numbers if membership funciton is given by

$$
\mu_{\mathrm{A}}(\mathrm{x})= \begin{cases}\frac{x-a}{b-a} & \mathrm{a} \leq \mathrm{x} \leq \mathrm{b} \\ 1 & \mathrm{~b} \leq \mathrm{x} \leq \mathrm{c} \\ \frac{x-d}{c-d} & \mathrm{c} \leq \mathrm{x} \leq \mathrm{d}\end{cases}
$$

\subsection{Ranking function: [defuzzified values]}

A convenient method for using fuzzy numbers is by Ranking [5],[6] (centre of Area) It is defined by $\mathbf{R}(\breve{\mathbf{A}})=J_{x} x \mu_{\check{A}}(\mathrm{x})$ $\mathrm{d} x$

$$
\int_{x} \mu_{\check{A}}(x) d x
$$

where $\mu_{\mathrm{A}}(\mathrm{x})$ is membership function (aggregated) and $\mathrm{x}$ is the output variable.

\section{Method of Ranking (Centroid of Area)}

The following is the procedure for finding Ranking function for the given Trapezoidal fuzzy numbers $(a, b, c, d)$.

$$
\begin{aligned}
& \int \mu_{\mathrm{A}}(\mathrm{x}) \mathrm{d} \mathrm{x}=\int_{a}^{b} \frac{x-a}{b-a} d x+\int_{b}^{c} d x+\int_{c}^{d} \frac{x-d}{c-d} \mathrm{dx} \\
& \int \mu_{\mathrm{A}}(\mathrm{x}) \mathrm{d} \mathrm{x}=\frac{1}{b-a}\left[\frac{(x-a)^{2}}{2}\right]+[c-b]+b \frac{1}{c-d}\left[\frac{(x-d) 2}{2}\right] \\
& =\frac{1}{2}[\mathrm{~d}+\mathrm{c}-\mathrm{b}-\mathrm{a}] \\
& \int \mathrm{x} \mu_{\mathrm{A}}(\mathrm{x}) \mathrm{d} \mathrm{x}=\int_{a}^{b} x \frac{x-a}{b-a} d x+\int_{b}^{c} x d x+\int_{c}^{d} x \frac{x-d}{c-d} d x \\
& =\frac{1}{b-a}\left[\frac{(x-a)^{s}}{3}+a \frac{(x-a)^{2}}{2}\right]_{a}^{b}+ \\
& {\left[\frac{\left(c^{2}-b^{2}\right)}{2}\right]+\frac{1}{c-d}\left[\frac{(x-d)^{s}}{3}+d \frac{(x-d)^{2}}{2}\right]_{c}^{\mathrm{d}}} \\
& =\frac{1}{6}\left[d^{2}+c^{2}-b^{2}-a^{2}-a b+c d\right]
\end{aligned}
$$$$
\text { Therefore } \mathbf{R}(\breve{\mathbf{A}})=\quad \int_{\mathrm{x}} \mathrm{x} \mu_{\check{\mathrm{A}}}(\mathrm{x}) \mathrm{d} \mathrm{x}
$$$$
\int_{x} \mu_{\check{A}}(x) d x
$$$$
=(1 / 3\{[(a+b+c+d)-(c d-a b) /[(c+d)-(a+b)]\}
$$

Special Case:

When $\mathrm{b}=\mathrm{a} \mathbf{R}(\breve{\mathbf{A}})=(\mathbf{a}+\mathbf{b}+\mathbf{d}) / \mathbf{3}$ 


\section{International Journal of Science and Research (IJSR) \\ ISSN (Online): 2319-7064}

Index Copernicus Value (2015): 78.96 | Impact Factor (2015): 6.391

\section{Description of the Model}

Trapezoidal fuzzy numbers are converted into expected time (Normal time) by using method of Ranking.. These values treated as a normal time between the nodes and Shortest Path is calculated by using the given algorithm.

\subsection{Algorithm}

Step 1: Construct the network according to Fulkerson's Rule.

Step 2: From given the Trapezoidal fuzzy numbers using the method of Ranking function find the normal time.

Step 3: Find the number of possible ways (path from initial node to end)

Step 4: From the all possible ways find the summation of normal time, from that find the minimum distance from initial node to terminal node .[ie] Shortest path to complete the project

\section{Numerical Example}

Consider a small network with 4 notes and 5 activities. The distance between them is represented by Trapezoidal fuzzy Nos.

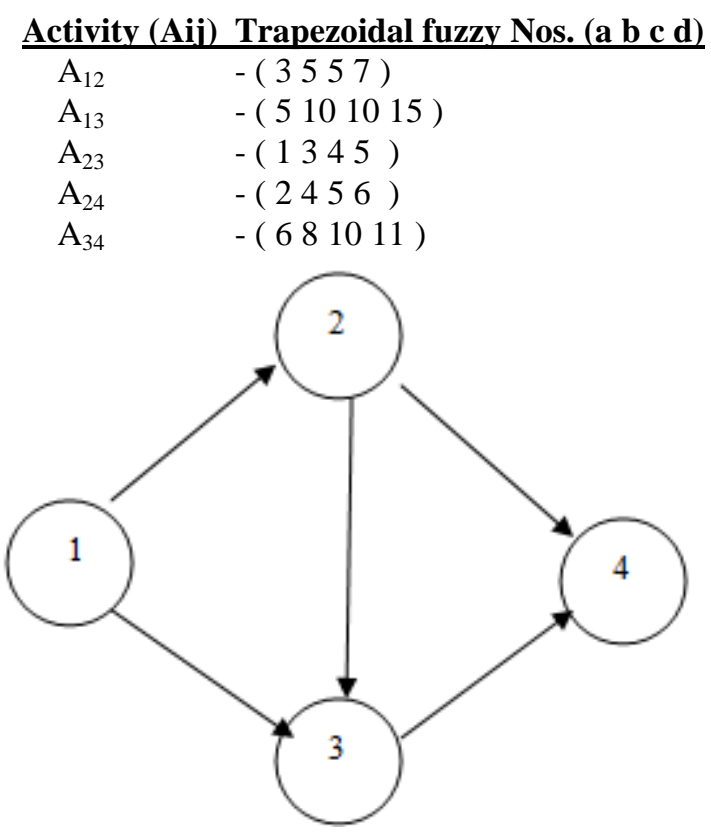

Stage 1: After evaluating the normal time by the method of Ranking function we obtain.

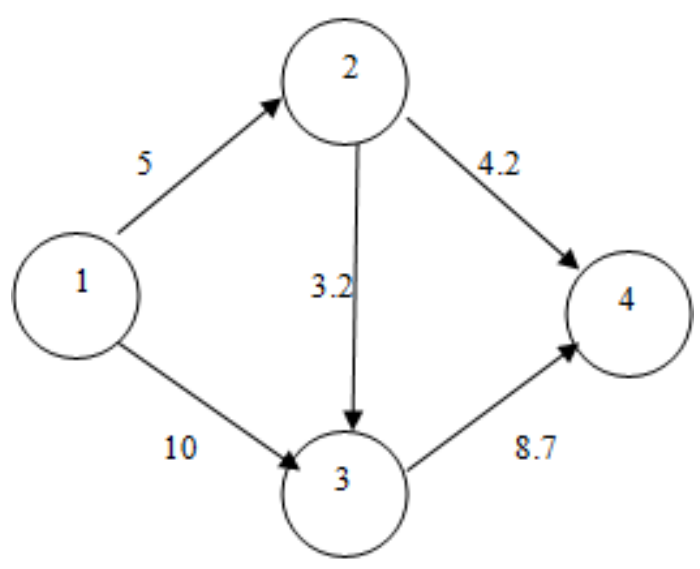

Stage 2: The possible ways are

$$
\begin{aligned}
& 1-2-4=9.2, \\
& 1-2-3-4=16.9, \\
& 1-3-4=18.7
\end{aligned}
$$

\section{Conclusion}

In this paper Ranking Function formula applied for trapezoidal fuzzy numbers to convert into normal time and applied given algorithm to find the shortest path from the initial vertex to end vertex.. The decision maker to decide the best possible shortest path in fuzzy environments.

\section{References}

[1] Kiranyadav .B RanjitBiswa, 2009. Finding a Shortest Path using an Intelligent technique. Int. Jnl. of engg. Tech., 1(2): 1793-8326.

[2] A. NagoorGani and M. Mohamed Jabarulla. 2010. On Searching Intutiionistic Fuzzy shortest path in a Network. Applied Mathematical science (29): 34473454.

[3] D. Dubois ad H Prade 1980 Fuzzy Sets and systems Theory and Applns. Academic Press, New York.

[4] Yamakami A The Shortest Path Problem on Network with fuzzy parameters, Fuzzy sets and systems 15815611570, 2007.

[5] Salimrezvani. Ranking method of Trapezoidal Intuitionitic fuzzy, annals of fuzzy mathematics and informatics, Vol 5 No 3(2013)

[6] J.S.Yao and K.M Wu 2000 Ranking fuzzy numbers based on decomposition principles and signed distance . Fuzzy sets and systems 116' 275 -288

[7] K.C Lin and M.S. Chem .1993. The fuzzy shortest path problem and its most vital areas .fuzzy sets and systems. $58(3) ; 343-353$

[8] P.K De and AmtaBhincar .2010 Computation of shortest path in fuzzy network. Intl Jrnl of computer applns. 11(2) $0975-887$

[9] R.SophiaPorchelvi and G.Sudha 2013 .A modified algorithm of solving shortest path problem with intuitionistic fuzzy arc lengths . Intl Jrnl of scientific and Engg. Research. 4(10);2229-5518

[10]Liu S.T and Kao .C .2004 Network flow problem with fuzzy area length . IEEE transaction on systems manandcybermetics Part B 34; 765-769 